\title{
A Three-Parameter Langmuir-Type Model for Fitting Standard Curves of Sandwich Enzyme Immunoassays with Special Attention to the $\alpha$-Fetoprotein Assay
}

\author{
W. KortlandT, H. J. ENDEMAN, AND J. O. O. HoEKE \\ University IIospital, Department of Clinical Biochemistry, Utrecht, The Netherlands
}

Received April 3, 1986

\begin{abstract}
In a simplified approach to the reaction kinetics of enzyme-linked immunoassays, a Langmuir-type equation $y=[a x /(b+x)]+c$ was derived. This model proved to be superior to logit-log and semilog models in the curve-fitting of standard curves. An assay for $\alpha$-fetoprotein developed in our laboratory with a sensitivity of $2 \mathrm{~g} /$ liter and a between-day coefficient of variation of about $15 \%$ was used for comparison of the different models. The proposed model has the advantage that it is based on the law of mass action and that blank determinations can be included in the calculation of the curves without giving a disproportionate bias. (C) 1987 Academic Press, Inc.

KEY WORDS: binding assays; clinical chemistry; immunochemical methods; cancer chemotherapy; statistics.
\end{abstract}

Since the introduction of the radioimmunoassay (RIA) ${ }^{1}$ numerous articles on reaction kinetics as well as on the best fit of dose-response curves have been published (1-6). However, a number of immunological techniques developed later obey different reaction kinetics from the classical RIA. Some of these methods, like the immunosorbent assays, are based on the phenomenon that immunoglobulins adsorb to all kinds of surfaces. The introduction of enzyme labels adds another dimension. Curve-fitting procedures for such techniques are rarely investigated. An example of such a method is the enzyme-linked immunosorbent assay (ELISA) (7). Most authors using ELISA techniques still plot their standard curves by hand on semilog paper, resulting in beautiful sigmoids, they perform a linear logit-log

\footnotetext{
${ }^{1}$ Abbreviations used: RIA, radioimmunoassay; ELISA, enzyme-linked immunosorbent assay; AFP, $\alpha$-fetoprotein; PBS, phosphate-buffered saline; CV, coefficient of variation; MSQ, mean sum of squares; IRMA, immunoradiometric assay.
}

curve-fitting procedure analogous to the RIA procedure, or they do a semilogarithmic regression analysis (8). Apart from his numerous papers on the radioimmunoassay, Rodbard $(9,10)$ also investigated the basis of the kinetics of a comparable assay, the immunoradiometric assay. However, no simple curve-fitting can be performed based on his four-parameter model.

Here we present a simple three-parameter equation that can be derived from the law of mass action and that can be applied well to sandwich assays. Using this equation we calculated results obtained with our sandwich ELISA for $\alpha$-fetoprotein and compared them with figures obtained by linear logit-log curve-fitting and quadratic semilog curve fitting.

\section{THEORETICAL DERIVATION}

In a simplified approach to the reactions involved in sandwich enzyme-linked immunoassays, we considered three reactions to be of major importance: first, a reaction of 
coated antibody $Q$ with antigen $P$, second, the reaction of complex $Q P$ with a second labeled antibody $Q^{\circ}$; and third, the reaction of the complex $Q P Q^{\circ}$ with a substrate resulting in a detectable product. We disregarded the possible dissociation of complexes during washing procedures between incubations and thereafter.

$$
\begin{aligned}
Q+P & \rightleftharpoons Q P \\
Q P+Q^{\circ} & \rightleftharpoons Q P Q^{\circ} \\
Q P Q^{\circ}+\text { substrate } & \rightleftharpoons \text { product }(y) .
\end{aligned}
$$

If the initial concentrations of $P$ and $Q$ are $p$ and $q$, then equilibrium [E.1] leads to

$$
K=\frac{(q-Q P) \cdot(p-Q P)}{Q P}
$$

or

$$
Q P^{2}-(q+p+K) \cdot Q P+q \cdot p=0 .
$$

Assuming that $K \gg q$ (or $p \gg q$ or $q \gg p$ ) it follows that $Q P \ll p$. Then $Q P$ can be approximated as

$$
Q P=\frac{q \cdot p}{(q+p+K)} .
$$

Equilibrium [E.2] gives an equation similar to [A.1]. If we assume excess $Q^{\circ}$, then the following formula holds:

$$
Q P Q^{\circ}=\frac{Q^{\circ} \cdot Q P}{K^{\prime}+Q^{\circ}}
$$

In the presence of excess substrate the reaction product ( $y$ ) of $Q P Q^{\circ}$ is related linearly to the concentration of $Q P Q^{\circ}$. Substituting [A.2] in [A.3] and using $f=q \cdot Q^{\circ} /\left(k^{\prime}+Q^{\circ}\right)$ times a constant in which reaction time, temperature, and enzyme activity are included, the following formula results:

$$
y=\frac{f \cdot p}{q+p+K} .
$$

As the quantity of coated antibody is constant within each assay, we can substitute a new constant $g$ for $(q+K)$. Also a blank correction $h$ can still be added to this formula, resulting in our working model:

$$
y=\frac{f \cdot p}{g+p}+h
$$

The commonly used logit-log curve model can be related to our model [A.5]. To show this, [A.4] must first be written as

$$
\frac{y}{f-y}=\frac{p}{g} \text {. }
$$

A so-called index of heterogeneity $(i)$ to correct for nonuniformity of the antibody (11) is now introduced. After logarithmic transformation, this results in the following logit-log form, which we compared with our model [A.5]:

$$
\log \frac{y}{f-y}=i \log p-i \log g .
$$

No blank correction is made because this would introduce a fourth parameter. The third equation comes from the linear regression of the response versus the log dose sometimes used (8). There is no theoretical background for this solution. To fulfill the three parameters we compared the quadratic expression

$$
y=a(\log p)^{2}+b \log p+c .
$$

\section{MATERIALS AND METHODS}

The AFP assay was performed essentially as described by Macdonald (12). Polystyrene microtiter plates were obtained from Greiner, Alphen a/d Rijn, The Netherlands (art. 655101). The plates were coated with a $1: 1000$ concentration of porcine anti-human AFP serum (Orion Diagnostica, Helsinki; Catalog No. D-752) in $125 \mathrm{mM}$ carbonate buffer (pH 9.6).

Calibration curves were composed of AFP standard (Behringwerke, West Germany) diluted in pretreated rabbit serum $(30 \mathrm{~min}$ at $56^{\circ} \mathrm{C}$ ) to eight concentrations ranging from 2 to $400 \mu \mathrm{g} /$ liter. Peroxidase-conjugated rabbit immunoglobulin to human AFP, obtained from Dakopatts, Sweden (Code P 128), was used at a 1:1000 dilution in phosphate-buffered saline (PBS). The sample and conjugate 
TABLE 1

Median ABSORbance, AVERAge Standard DeVIATION AND AVERAGE COEFFICIENT OF VARIATION AS A FUNCTION OF AFP CONCENTRATION

\begin{tabular}{cccc}
$\begin{array}{c}\text { Concn. } \\
\text { (mg/liter) }\end{array}$ & $\begin{array}{c}\text { Absorption } \\
\text { (median) }\end{array}$ & $\begin{array}{c}\text { SD } \\
\text { response }\end{array}$ & $\begin{array}{c}\text { CV } \\
\text { response }\end{array}$ \\
\hline 0 & 191 & 14.0 & 6.7 \\
2 & 207 & 15.9 & 6.6 \\
4 & 249 & 15.6 & 5.8 \\
10 & 377 & 15.7 & 4.0 \\
20 & 490 & 16.2 & 3.3 \\
40 & 663 & 23.0 & 3.6 \\
100 & 922 & 30.0 & 3.3 \\
200 & 1122 & 33.4 & 3.0 \\
400 & 1205 & 38.8 & 3.1 \\
\hline
\end{tabular}

incubations were performed at $37^{\circ} \mathrm{C}$ for 30 and $60 \mathrm{~min}$, respectively. Between the incubations the plates were washed three times with PBS containing $5 \%$ rabbit serum and $0.1 \%$ Tween 20. Color development was initiated by adding OPD reagent at $\mathrm{pH} 5.5$ and subsequently stopped after 30 min with $4 \mathrm{~N}$ $\mathrm{HCl}$. Measurements were taken with an ELISA reader (Titertek) at $492 \mathrm{~nm}$.

Least-squares curve-fitting by means of the Gauss-Newton iteration method was done on an HP-85 desktop computer. Blank measurements were not included in the fitting procedures. Statistical analyses were performed according to standard methods (13-14). The within-assay precisions were calculated from the standard deviations of the standards ( $n=3$ or 4 for each standard within each assay). The mean within-assay precision was averaged over several assays.

\section{RESULTS}

The test parameters of the $\alpha$-fetoprotein assay we developed are comparable to other ELISA's for $\alpha$-fetoprotein $(12,15)$ and are given below.

The sensitivity of the assay was $2 \mu \mathrm{g} /$ liter (corresponding to a response of twice the standard deviation above the mean blank).
The within-assay coefficient of variation (CV) is calculated using the standards of the dose-response curve (Table 1). Based on these figures a precision profile according to Ekins and Edwards (16) can be calculated, as shown in Fig. 1.

The between-day $\mathrm{CV}$ was tested over a year with two frozen samples $\left(-20^{\circ} \mathrm{C}\right)$ at different concentrations. This resulted in a CV of $13 \%$ at a level of $18.8 \mu \mathrm{g} / \mathrm{liter}(n=28)$ and a CV of $16 \%$ at a level of $95 \mu \mathrm{g} / \mathrm{liter}(n=37)$.

Dose-response curve data for 47 assays were fitted according to three models (Eqs. [A.5], [A.6], and [A.7]). To compare these models we used the mean sum of squares (MSQ). According to this parameter, Eq. [A.5] nearly always fit better than [A.6] (in 44 out of 47 assays) and Eq. [A.6] in most cases fit better than Eq. [A.7] (41 out of 47; see Table 2). Although the differences between the MSQs were significant in only a few individual assays using a Student $t$ test (see Table 2), the difference between the MSQ of Eq. [A.5] and that of the other two models was highly significant $(p \ll 0.001, n$ $=47$ ) when all assays were taken into account. Likewise, Eq. [A.6] fit significantly better than [A.7] $(p \ll 0.001, n=47)$. The mean parameters of all curves and their standard deviations are shown in Table 3.

Figure 2 shows a typical example of a dose-response curve. The computer fits of both [A.5] and [A.6] are shown.

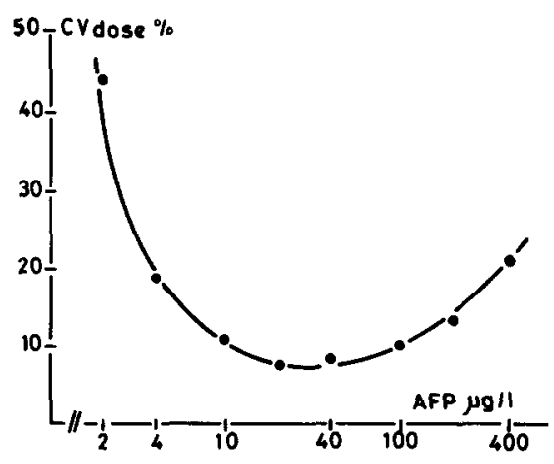

FIG. 1. Precision profile of the AFP assay. 
TABLE 2

NUMBER OF INDIVIDUAL ASSAYS IN WHICH THE CURVE-FITTING ACCORDING TO EQ. [A.5], [A.6], or [A.7] RESULTED IN A HIGHER AND RESPECTIVELY LoWer MeaN SUM of SQUARES

\begin{tabular}{|c|c|c|c|c|}
\hline \multirow{2}{*}{\multicolumn{2}{|c|}{$\begin{array}{l}\text { Mean } \\
\text { sum of } \\
\text { squares }\end{array}$}} & \multicolumn{3}{|c|}{ Larger } \\
\hline & & [A.5] & [A.6] & [A.7] \\
\hline \multirow{3}{*}{ Smaller } & [A.5] & & $44(6)$ & $46(11)$ \\
\hline & [A.6] & 3 & & 41 \\
\hline & [A.7] & 2 & $6(1)$ & \\
\hline
\end{tabular}

Note. The number of assays which differed significantly at the $10 \%$ level by the $F$ test is shown in parentheses.

\section{DISCUSSION}

The calibration curves were handdrawn initially in our laboratory but in order to eliminate any arbitrariness we looked for a mathematical model with the least number of parameters that would result in a good fit. We tried the logit-log fit, very commonly used in radioimmunoassays, the less common semilog fit (both used in some ELISA kits (8)), and a fit of a Langmuir-type equation.

A very important disadvantage of the logit-log model is the high weight that is assigned to the blank measurement, which in most instances is far from accurate. Furthermore, values of saturating high antigen concentrations are unreliable because of high dose hook effects which may be present.

Very good fits were obtained with the Langmuir-type equation [A.5] and, making some rough approximations, this equation could be derived from the law of mass action, as shown in the theoretical part of this paper. We assumed the coated antibody $Q$ to be in free solution so we could work in terms of concentrations. Based on a completely different model-lattices of immobilized binding sites-Pincus and Rendell (17) derived equations similar to ours, which are based on concentrations only. The resulting equation is essentially the same as conventional equa- tions especially where they assume that the added substance binds preferentially to only one binding site and that no binding sites are excluded. Therefore we think that our assumption about concentrations is a reasonable approximation. Also we neglected minor reactions. As our experimental doseresponse curves nearly always fit this model very well, these assumptions appear to be justified.

The parameter $g$ of Eq. [A.5] is equal to $q$ $+K$ and is estimated to be $65 \mathrm{ng} / \mathrm{ml}$ (Table 3) or $0.94 \pm 0.20 \mathrm{nmol} /$ liter. In order to check whether $K \gg q$ (the most important limiting condition, especially in the middle of the curve, when $p \approx q$ ) we can estimate the adsorbed quantity of specific anti-AFP immunoglobulin. Cantarero et al. (19) have reported experiments on the adsorption characteristics of some proteins to polystyrene. He noted that albumin and IgG immunoglobulins are adsorbed up to about 150 $\mathrm{ng} / \mathrm{cm}^{2}$. If we assume that about 1 to $2 \%$ of our coating serum consists of specific anti$\alpha$-fetoprotein (Gmelig-Meyling, personal communication), then there will be about $2.3 \mathrm{ng} / \mathrm{cm}^{2}$ available for AFP binding, which corresponds to $0.16 \mathrm{nmol} / \mathrm{liter}\left(M_{\mathrm{r}}\right.$ of $\mathrm{IgG}$ is 150,000 and a $100-\mu 1$ incubation volume corresponds to $0.95 \mathrm{~cm}^{2}$ in our assay procedure). We now get an estimate of $K$ being $0.78 \pm 0.21 \mathrm{nmol} /$ liter. The assumption $K$

TABLE 3

MEAn PaRameters of DOSE-Response CuRves [A.5], [A.6], and [A.7] AND THEIR STANDARD DEVIATIONS

\begin{tabular}{cccc}
\hline & \multicolumn{3}{c}{ Equation } \\
\cline { 2 - 4 } Parameter & {$[$ A.5] } & {$[$ [A.6] } & [A.7] \\
\hline$f, f, a$ & $1167 \pm 203$ & $2001 \pm 457$ & $21.8 \pm 6.4$ \\
$g, i \log g, b$ & $65 \pm 14$ & $2.66 \pm 0.33$ & $51.4 \pm 44.5$ \\
$h, i, c$ & $202 \pm 77$ & $0.53 \pm 0.09$ & $163 \pm 68$ \\
\hline
\end{tabular}

Note. The parameters of [A.6] and [A.7] are based on natural logarithms. 


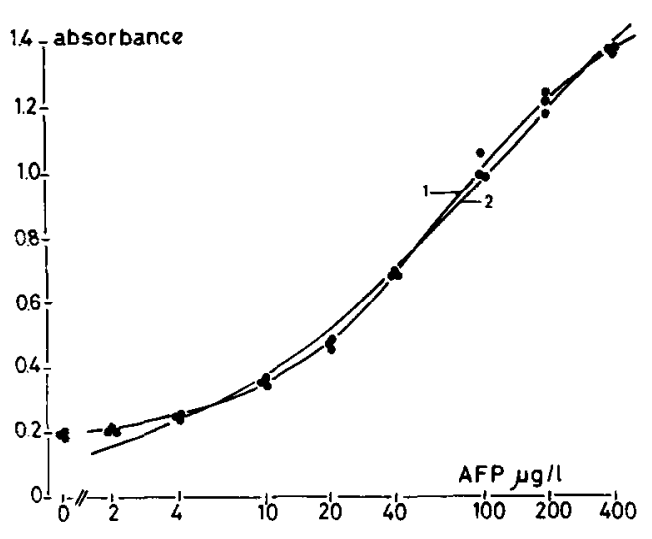

FIG. 2. Dose-response curves of the AFP assay. Curve-fitting was performed using (1) Eq. [A.5] and (2) Eq. [A.6].

$\gg q$ is not completely satisfied. However, based on the estimates of $q$ and $K$, we calculated that Eq. [A.5], when fitted by least squares, gave a solution to the basic Eq. [A.1] in which the bias of the response is less than $2 \%$.

The other two models tested resulted in inferior fits. The semilog form has no theoretical background. The logit-log form (Eq. [A. 6]) has some; however, no corrections for the blank are included in this cquation.

Introduction of a blank parameter results in a four-parameter equation which has the same characteristics as the empirical four-parameter logistic equation ${ }^{2}$

$$
y=\frac{a-d}{1+(x / c)^{b}}+d
$$

of Rodbard and Feldman (9). Recently the International Atomic Energy Agency convened a group which published general guidelines for all kinds of data processing in immunoassays (18). In this paper the fourparameter logistic is recommended. We tested the logistic model but although the fit was the same or slightly better than that for Eq. [A.5] for all individual calibration

\footnotetext{
${ }^{2}$ Parameters $f, g$, and $h$ in formula [A.5] correspond, respectively, to the parameters $-(a-d), c$, and $a$, with $b$ $=1$, in Rodbard et al. (9).
}

curves, the differences in the mean sum of squares were very small ( $\leqq 2.9 \%)$. Thus, the index of heterogeneity was calculated to be $i$ $=0.995 \pm 0.046(\mathrm{CV}=5 \% ; n=45)$. This index, not being different from $1(p>0.1)$, implies that the four-parameter function does not result in an improved fit when compared to our proposed three-parameter function [A.5] so our equation is preferred. The three-parameter function as a simplification of the logistic one is also mentioned by Munson (20) in a paper in which he compared several RIA calibration curve models. The three-parameter function there too displayed one of the best fits to the data.

Based on our data, Eq. [A.5] not only is tailor-made for this particular assay but also is applicable to most sandwich assays based on the same principle, e.g., ELISA and IRMA. We tested this for some recently published assays in which the dose-response curves (21-26) were shown. Five out of six different dose-response curves resulted in a better fit using Eq. [A.5] than when using Eq. [A.6]. Although in these particular assays the proposed three-parameter model can suffice, it may sometimes be necessary to fit data according to the four-parameter logistic (9).

In conclusion, the relatively simple threeparameter equation

$$
y=\frac{f \cdot x}{g+x}+h
$$

as a model for dose-response curve-fitting in sandwich assays gives a better result than some other models used in practice. It has some theoretical background, and, although the limiting conditions are not always completely met, the equation is suitable based on empirical data. Iterative calculations must be performed, but this is not a problem today as it can be done on even inexpensive computers.

\section{REFERENCES}

1. Berson, S. A., and Yalow, R. S. (1959) J. Clin. Invest. 38, 1996.

2. Ekins, R., Newman, B., and O'Riordan, J. L. H. 
(1968) in Radioisotopes in Medicine, in Vitro Studies, Vol. 13, p. 59-97, USAEC, Oak Ridge, TN.

3. Feldman, H., and Rodbard, D. (1971) in Principles of Competitive Protein-Binding Assays (Odell, W. O., and Doughaday, W. H., Eds.), pp. 158-203, Lippincott, Toronto.

4. Finney, D. J. (1983) Clin. Chem. 29, 1762-1766.

5. Raab, G. M. (1983) Clin. Chem. 29, 1757-1761.

6. Rodbard, D., Munson, P. J., and Delean, A. (1978) in Radioimmunoassay and Related Procedures in Medicine, Vol. 1, pp. 469-514, Intern. At. Energy Agency, Vienna.

7. Schuurs, A. H. W. M., and van Weemen, B. K. (1980) J. Immunoassay 1, 229-249.

8. Walker, R. O., and Uddin, Z. (1983) Clin. Chem. 29, 727-728.

9. Rodbard, D., and Feldman, Y. (1978) Immunochemistry 15, 71-76.

10. Rodbard, D., and Hutt, D. M. (1974) in Radioimmunoassay and Related Procedures in Medicine, Vol. 1, pp. 165-189, Internal Atomic Energy Agency, Vienna.

11. Butler, J. E. (1980) in Enzyme-Immunoassay (Maggio, E. T., ed.), p. 1, CRC Press, Boca Raton, FL.

12. Macdonald, J. E., and Kelly, A. M. (1978) Clin. Chim. Acta 87, 367-372.

13. Snedecor, G. W., and Cochran, W. G. (1979) Statistical Methods, 6th ed., Iowa State Univ. Press, Ames.

14. Green, J. R., and Morgerison, D. (1977) Statistical Treatment of Experimental Data, Elsevier, Amsterdam/New York.
15. Weeks, I., Campbell, A. K., and Woodhead, J. S. (1983) Clin. Chem. 29, 1480.

16. Ekins, R. P., and Edwards, P. R. (1983) in Immunoassays for Clinical Chemistry (Hunter, W. M., and Corrie, J. E. T., Eds.), pp. 76-105, Churchill Livingstone, Edinburgh.

17. Pincus, M. R., and Rendell, M. (1981) Proc. Natl. Acad. Sci. U.S.A. 78, 5924-5927.

18. Dudley, R. A., Edwards, P., Ekins, R. P., Finney, D. J., McKenzie, I. G. M., Raab, G. M., Rodbard, D., and Rodgers, R. P. C. (1985) Clin. Chem. 31, 1264-1271.

19. Cantarero, L. A., Butler, J. E., and Osborne, J. W. (1980) Anal. Biochem. 105, 375-382.

20. Munson, P. J. (1984) in Proceedings, American Statistical Association 1984, Biopharmaceutical Section, pp. 46-52, Amer. Statistical Assoc., Washington, DC.

21. Endo, Y., Yamamoto, I., Ohtaki, S., Yoshitake, S., and Ishikawa, E. (1983) Ann. Clin. Biochem. 20, 163-168.

22. Imagawa, M., Hashida, S., Ohta, Y., and Ishikawa, E. (1984) Ann. Clin. Biochem. 21, 310-317.

23. Yoshitaken, S., Endo, Y., Nakagawa, M., Imagawa, M., Ohtaki, S., and Ishikawa, E. (1982) Clin. Chim. Acta 125, 1-7.

24. Weeks, I., and Woodhead, J. S. (1984) Clin. Chim. Acta 141, 275-280.

25. Kato, K., Shimizu, A., Ishiguro, Y., Mokuno, K., Ariyoshi, Y., and Nakajima, T. (1985) Clin. Chim. Acta 150, 31-40.

26. Haimoto, H., and Kato, K. (1986) Clin. Chim. Acta 154, 203-212. 